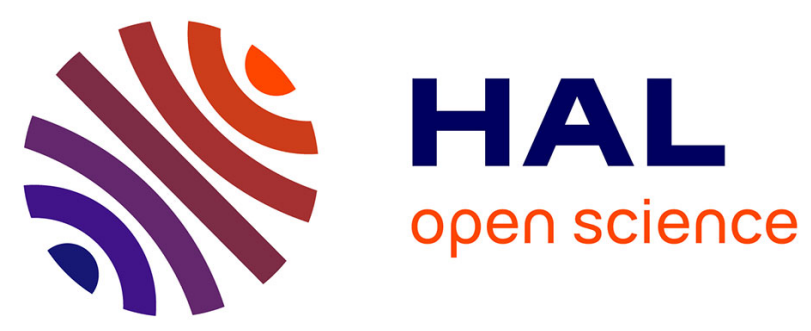

\title{
The importance of monopole antennas for dust observations: Why Wind/WAVES does not detect nanodust
}

\author{
N. Meyer-Vernet, M. Moncuquet, K. Issautier, A. Lecacheux
}

\section{- To cite this version:}

N. Meyer-Vernet, M. Moncuquet, K. Issautier, A. Lecacheux. The importance of monopole antennas for dust observations: Why Wind/WAVES does not detect nanodust. Geophysical Research Letters, 2014, 41 (8), pp.2716-2720. 10.1002/2014GL059988 . hal-02544414

\author{
HAL Id: hal-02544414 \\ https://hal.science/hal-02544414
}

Submitted on 7 Jan 2022

HAL is a multi-disciplinary open access archive for the deposit and dissemination of scientific research documents, whether they are published or not. The documents may come from teaching and research institutions in France or abroad, or from public or private research centers.
L'archive ouverte pluridisciplinaire HAL, est destinée au dépôt et à la diffusion de documents scientifiques de niveau recherche, publiés ou non, émanant des établissements d'enseignement et de recherche français ou étrangers, des laboratoires publics ou privés. 


\section{Geophysical Research Letters}

\section{RESEARCH LETTER \\ 10.1002/2014GL059988 \\ The importance of monopole antennas for dust observations: Why Wind/WAVES does not detect nanodust}

Key Points:

- We explain why Wind/WAVES cannot detect nanodust

- We propose a new technique for dust detection with dipole antennas

- We propose a novel interpretation of Wind/WAVES dust data

Correspondence to:

N. Meyer-Vernet,

Nicole.Meyer@obspm.fr

\section{Citation:}

Meyer-Vernet, N., M. Moncuquet, K. Issautier, and A. Lecacheux (2014), The importance of monopole antennas for dust observations: Why Wind/WAVES does not detect nanodust, Geophys. Res. Lett., 41, 2716-2720, doi:10.1002/2014GL059988.

Received 21 MAR 2014 Accepted 9 APR 2014 Accepted article online 13 APR 2014 Published online 24 APR 2014

\author{
N. Meyer-Vernet ${ }^{1}$, M. Moncuquet ${ }^{1}$, K. Issautier ${ }^{1}$, and A. Lecacheux ${ }^{1}$ \\ ${ }^{1}$ LESIA, CNRS, Observatoire de Paris, UPMC, Université Paris Diderot, Meudon, France
}

Abstract The charge released by impact ionization of fast dust grains impinging on spacecraft is at the basis of a well-known technique for dust detection by wave instruments. Since most of the impact charges are recollected by the spacecraft, monopole antennas generally detect a much greater signal than dipoles. This is illustrated by comparing dust signals in monopole and dipole modes on different spacecraft and environments. It explains the weak sensitivity of Wind/WAVES dipole antennas for dust detection, so that it is not surprising that this instrument did not detect the interplanetary nanodust discovered by STEREO/WAVES. We propose an interpretation of the Wind dust data, elsewhere discussed by Malaspina et al. (2014), which explains the observed pulse amplitude and polarity for interstellar dust impacts, as well as the nondetection of nanodust. This proposed mechanism might be the dominant dust detection mechanism by some wave instruments using dipole antennas.

\section{Introduction}

When a dust grain impacts a spacecraft at high speed, the electric charges produced by impact ionization induce an electric pulse which can be detected by onboard wave instruments. This technique was pioneered when the Voyager spacecraft crossed Saturn ring plane and the onboard radio (planetary radio astronomy (PRA)) [Aubier et al., 1983] and plasma wave (PWS) [Gurnett et al., 1983] instruments detected micron-sized dust grains. These instruments detected signals of very different amplitudes because they had different wave capture capabilities: PRA used the antennas as monopoles, whereas PWS used them as dipoles (Figure 1) - a difference which must be taken into account in analyzing the signals.

This serendipitous discovery opened the way to a new technique which was later used to measure micron-sized grains on Voyager in the dust rings of Uranus [Meyer-Vernet et al., 1986a; Gurnett et al., 1987] and Neptune [Gurnett et al., 1991; Pedersen et al., 1991], in cometary environments on Vega [e.g., Oberc, 1996] and ISEE-3/ICE [Gurnett et al., 1986; Meyer-Vernet et al., 1986b], in Saturn's E ring with Cassini [e.g., Kurth et al., 2006; Moncuquet and Schippers, 2013], in the solar wind near 1 AU with STEREO [Zaslavsky et al., 2012; Belheouane et al., 2012] and even in the outer solar system [Gurnett et al., 1997]. The technique was recently extended to measure fast nanodust in Jovian nanodust streams with Cassini [Meyer-Vernet et al., 2009b] and led to the discovery by STEREO of interplanetary nanodust picked up by the solar wind [Meyer-Vernet et al., 2009a; Zaslavsky et al., 2012; Le Chat et al., 2013].

Malaspina et al. [2014] have recently discussed the voltage pulses produced on Wind/WAVES by dust impacts. The nondetection by this instrument of the fast nanodust discovered by STEREO/WAVES led these authors to suggest an inconsistency between the STEREO and Wind results on nanodust, arguing that both spacecraft were in close proximity and had similar electric field waveform capture capabilities.

We submit that this discrepancy between Wind and STEREO is not surprising. In addition to the very dissimilar antenna geometries, both instruments have completely different electric field waveform capture capabilities: indeed, STEREO can use the antennas as monopoles, whereas Wind only uses them as dipoles. Since this difference between dipoles and monopoles is sometimes ignored outside the radio astronomy community, producing confusion as in the case of the recent paper by Malaspina et al. [2014], it is worth discussing it first. We then propose an interpretation of the Wind dust data, which may be relevant with other wave instruments using dipole antennas. 


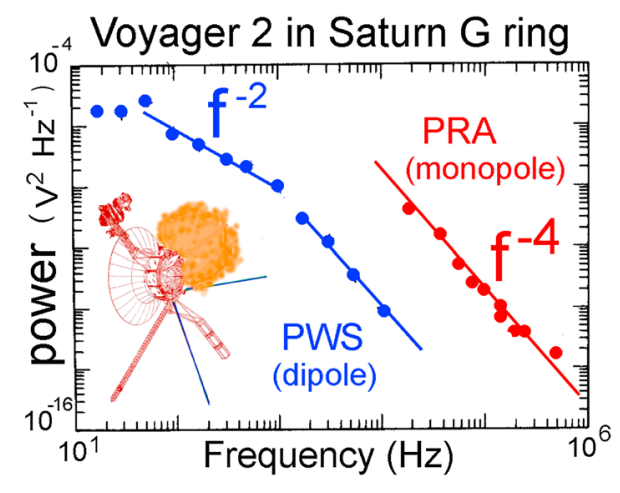

Figure 1. Voltage power spectrum measured in Saturn's $G$ ring by the (high-frequency) radio (PRA) and (lower frequency) plasma wave (PWS) instruments on Voyager 2, with respectively monopole and dipole antennas. At similar frequencies, the power is higher by nearly 4 orders of magnitude on the monopole than on the dipole. Adapted from Mann et al. [2011] with kind permission from Springer Science and Business Media.

\section{Dipole Versus Monopole Electric Antennas}

Since the spacecraft surface area generally exceeds that of the electric antennas by several orders of magnitude, most of the dust impacts occur on the spacecraft, which recollects most of the impact charges of sign opposite to its floating potential.

In dense planetary magnetospheres, the ambient plasma generally dominates the charging currents, producing a negative floating potential, whereas in the solar wind photoelectron emission dominates, producing a positive floating potential [e.g., Mann et al., 2014]. Therefore, in dense plasmas the target tends to recollect the positively charged ions of the impact plasma, whereas in the solar wind the target recollects the electrons. This produces a potential pulse on the target of the same sign as the recollected charges.

This mechanism produces a potential pulse $\delta V_{\mathrm{sc}} \sim Q / C_{\mathrm{sc}}$ on a spacecraft of capacitance $C_{\mathrm{sc}}$ recollecting the charge $Q$, so that each monopole antenna, say $y$ (detecting the potential between itself and the spacecraft structure) measures a pulse $\delta V_{y} \simeq-\delta V_{\text {sc }}$. In contrast, a dipole antenna

(detecting the potential between two arms, say $y_{+}$and $y_{-}$) measures via this mechanism a much smaller signal, produced by circuit imbalances (common mode rejection factor, differences in antenna, and base capacitances).

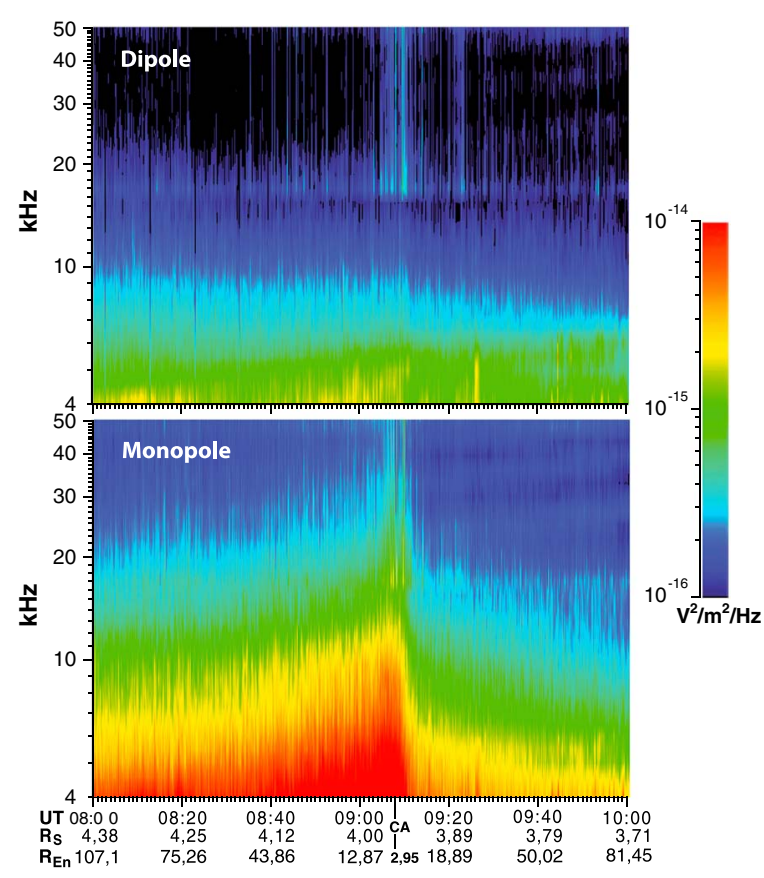

Figure 2. Time-frequency electric power spectral density measured by Cassini/RPWS between 4 and $50 \mathrm{kHz}$ during the first close approach of Enceladus (on 9 March 2005) with (top) dipole and (bottom) monopole antennas. Both spectrograms are calibrated in $V^{2} / \mathrm{m}^{2} / \mathrm{Hz}$ (color chart). The signal produced by $\mathrm{E}$ ring dust impacts is clearly seen in monopole mode near the closest approach of Enceladus orbit. The time and Cassini's distance from Saturn (in $R_{S}=60,330 \mathrm{~km}$ ) and from Enceladus (in $R_{\mathrm{En}}=252 \mathrm{~km}$ ) are indicated every $20 \mathrm{~min}$ at the bottom.
Figure 1 shows the power spectra detected by the (high-frequency) radio [Aubier et al., 1983] and by the (low-frequency) plasma wave instrument [Gurnett et al., 1983] on board Voyager 2 in Saturn's G ring, with monopole and dipole antennas, respectively. The power spectrum is proportional to the square of the pulse voltage amplitude produced by individual impacts and to the impact rate (see Meyer-Vernet [1985] for a detailed calculation of the spectral shape). One sees that the dipole antennas recorded a power smaller than the monopole by nearly 4 orders of magnitude, in agreement with the signal being mainly produced by recollection by the spacecraft of the impact charges produced by grain impacts on its surface [Meyer-Vernet, 1985; Oberc, 1996; Meyer-Vernet et al., 1996, 1998]. The smaller power spectrum observed in dipole mode can be due in particular to recollection by an antenna of a fraction of the charges produced by impacts on the spacecraft or on itself [Gurnett et al., 1983].

Figure 2 compares the electric power spectra measured by the Cassini/Radio and Plasma Wave Science (RPWS) 


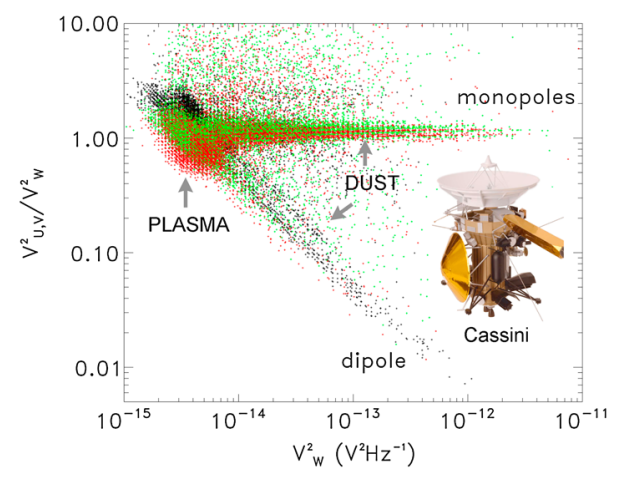

Figure 3. Cassini/RPWS high-frequency receiver data in the Jovian outer magnetosheath. Ratios of the power on two monopoles (red and green) to that on the other one as a function of the latter and ratio of the power on the dipole to that on the monopole (black). The dust impacts yield similar signals on each monopole, whereas the dipole records mainly the plasma thermal noise (of smaller amplitude). Adapted from Meyer-Vernet et al. [2009b].

essentially measures the weaker plasma quasi-thermal and impact shot noise calculated by Meyer-Vernet and Perche [1989].

On Voyager and Cassini, the electric antennas have a radius of about $1 \mathrm{~cm}$, and a surface area smaller than the spacecraft one by nearly 2 orders of magnitude. In contrast, the Wind/WAVES antennas are thin wires of $0.19 \mathrm{~mm}$ radius [Sitruk and Manning, 1997] extending perpendicular to the cylindrical spacecraft surface. Therefore, they are expected to collect a still smaller fraction of the impact released charges.

Could the voltage observed by Wind/WAVES be nevertheless produced by recollection by the antennas of the impact-produced charges, as is often assumed to explain the dust observations in dipole mode? This explanation, originally invoked by Malaspina et al. [2014], is inconsistent with the data since the floating potential of surfaces in the solar wind is positive, making them recollect electrons, rather than (positive) ions; hence, the recollection (of electrons) by an antenna arm should produce on it a negative potential pulse instead of a positive one. The voltage observed in dipole mode should then be of sign opposite to that required to yield the correct direction of interstellar dust flow.

\section{Alternative Detection Mechanism With Thin Dipole Antennas}

Therefore, impact charge recollection by the antennas cannot explain the voltage pulses measured by Wind/WAVES. The mechanism of destabilization of the photoelectrons surrounding the antennas suggested by Pantellini et al. [2012, 2013] and implemented by Zaslavsky et al. [2012] on STEREO requires antennas of large radius and an adequate geometry (the STEREO/WAVES antennas extend close to the spacecraft plane faces). Even if the geometry were adequate, the $0.19 \mathrm{~mm}$ radius of the Wind spin-plane dipoles would preclude this mechanism to be significant because the photoelectron current is too small [Pantellini et al., 2012]. What is then the origin of the pulses detected by the Wind dipole antennas?

We propose that they are produced by the electrostatic voltage induced on the antennas by the impact-produced positive ions after the spacecraft has recollected the electrons. This mechanism is consistent with the voltage sign observed on Wind for interstellar grain impacts, since the antenna arm closer to the impact site will then measure a larger positive voltage. Let us estimate the amplitude. The voltage produced on each antenna arm by a charge $Q$ can be calculated by averaging along the length of this antenna arm the Debye shielded Coulomb potential at distance $r, Q \times e^{-r / L_{D}} /\left(4 \pi \epsilon_{0} r\right), L_{D}$ being the Debye length; this holds for charges of speed much smaller than the electron thermal speed, which is the case for the impact-produced ions. Therefore, a cloud of electric charge $Q$ produces a voltage pulse of amplitude $\delta V \sim \alpha Q /\left(4 \pi \epsilon_{0} L\right)$ (in order of magnitude) on an antenna arm of physical length $L$ when it is closer to the antenna axis than the shielding Debye length (see Meuris et al. [1996] in another context). The value of $\alpha$, of order of magnitude unity, depends on the impact geometry, the spacecraft and antenna geometry, and the 
size and charge of the impact plasma cloud, with respect to the shielding capabilities of both the ambient plasma and the photoelectrons ejected by the spacecraft and antennas.

If the two antenna arms are separated by more than a Debye length, the arm closer to the impact site experiences a much higher voltage than the other one, so that the signal can be measured both in monopole and dipole modes with a similar order of magnitude; otherwise, the dipole voltage will be somewhat smaller, by an amount depending on the asymmetry of the impact with respect to the antennas. As noted above, the voltage sign agrees with the Wind dipole observations. This induced voltage is of the same sign as the voltage produced on a monopole antenna by charge recollection by the spacecraft, but generally much smaller (by the factor $\alpha C_{s c} / 4 \pi \epsilon_{0} L$ ), so that it may be barely seen with a monopole antenna. However, it may be the dominant detecting mechanism with thin dipole antennas having well-separated arms as in the case of Wind or in dense magnetospheres when photoelectron emission is negligible; in the latter case, since the nonrecollected charges would be the cloud's electrons, possibly moving faster than those of a cold ambient plasma, the calculation of the voltage is more complicated, possibly involving plasma waves.

Consider a grain of mass $m$ impacting at speed $v$, producing the impact charge $Q \simeq 0.7 \mathrm{~m}_{\mathrm{kg}}^{1.02} v_{\mathrm{km} / \mathrm{s}}^{3.48} \subset[M c B r i d e$ and McDonnell, 1999]. The above estimate yields a pulse of peak maximum amplitude

$$
\delta V \sim 0.7 \mathrm{~m}_{\mathrm{kg}}^{1.02} v_{\mathrm{km} / \mathrm{s}}^{3.48} \Gamma \alpha /\left(4 \pi \epsilon_{0} L\right) \text { volts }
$$

on an antenna arm of length $L$ (in $\mathrm{m}$ ) and receiver gain $\Gamma$.

With a $10 \mathrm{~nm}$ radius nanograin of mass $m \simeq 10^{-20} \mathrm{~kg}$ impacting at $300 \mathrm{~km} / \mathrm{s}$, as predicted by dynamics [Mann et al., 2014], $L \simeq 7.5 \mathrm{~m}$, and $\Gamma \simeq 0.4$ for the Wind/WAVES $E_{y}$ dipole [Sitruk and Manning, 1997], equation (1) yields a voltage pulse in the $\mathrm{mV}$ range, below the sensitivity of the instrument, and a still smaller voltage on the longer $E_{x}$ dipole.

In contrast, a grain of radius $1 \mu \mathrm{m}\left(\mathrm{m} \simeq 10^{-14} \mathrm{~kg}\right)$ impacting at about $30 \mathrm{~km} / \mathrm{s}$ should yield from (1) a voltage pulse on the Wind dipole antennas of order of magnitude $200 \mathrm{mV}$, which suggests that this mechanism is able to explain the range of amplitudes observed.

\section{Conclusions}

We conclude the following:

1. Our analysis taking into account the waveform capture capability of Wind/WAVES in dipole mode indicates that this instrument is unable to detect the interplanetary nanodust discovered by STEREO.

2. A new dust detection mechanism is proposed which should explain the amplitude and polarity of the voltage pulses observed on Wind when dust grains in the tenths of micron to micron size range impact the spacecraft.

3. This new mechanism may be the dominant dust detection mechanism by wave instruments using thin dipole antennas on other spacecraft; in particular, its applicability to the dipole observations on Voyager, Cassini and future spacecraft instrumentation, in addition to Wind, should be examined in detail.

Acknowledgments

The Cassini/RPWS HF receiver data are available at University of lowa and at LESIA (Observatoire de Paris, France). We thank the CNES and the CNRS for funding.

The Editor thanks two anonymous reviewers for their assistance in evaluating this paper.

\section{References}

Aubier, M. G., N. Meyer-Vernet, and B. M. Pedersen (1983), Shot noise and particle impacts in Saturn's ring plane, Geophys. Res. Lett., $10,5-8$.

Belheouane, S., et al. (2012), Detection of interstellar dust with STEREO/WAVES at 1 AU, Sol. Phys., 281, 501-506.

Graps, A. L., et al. (2001), lo Revealed in the Jovian Dust Streams, in Proceed. Meteor. 2001 Conf., ESA SP-495, edited by B. Warmbein, pp. 601-608, ESTEC, Nordwijk.

Gurnett, D. A., et al. (1983), Micron-sized particles detected near Saturn by the Voyager plasma wave instrument, Icarus, 53, 236-254.

Gurnett, D. A, T. F. Averkamp, F. L. Scarf, and E. Grün (1986), Dust particles detected near Giacobini-Zinner by the ICE plasma wave instrument, Geophys. Res. Lett., 13, 291-294.

Gurnett, D. A., et al. (1987), Micro-sized particle impacts detected near Uranus by the Voyager 2 plasma wave instrument, J. Geophys. Res., 92, 14,959-14,968.

Gurnett, D. A., W. S. Kurth, L. J. Granroth, and S. C. Allendorf (1991), Micro-sized particles detected near Neptune by the Voyager 2 plasma wave instrument, J. Geophys. Res., 96, 19,177-19,186.

Gurnett, D. A., J. A. Ansher, W. S. Kurth, and L. J. Granroth (1997), Micron-sized dust particles detected in the outer solar system by the Voyager 1 and 2 plasma wave instruments, Geophys. Res. Lett., 24, 3125-3128.

Gurnett, D. A., et al. (2004), The Cassini radio and plasma wave investigation, Space Sci. Rev., 114, 395-463.

Kurth, W. S., T. F. Averkamp, D. A. Gurnett, and Z. Wang (2006), Cassini RPWS observations of dust in Saturn's E ring, Planet. Space Sci., 54, 988-998. 
Le Chat, G., et al. (2013), Interplanetary nanodust detection by the Solar Terrestrial Relations Observatory/WAVES low frequency receiver, Sol. Phys., 286, 549-559.

Malaspina, D., et al. (2014), Interplanetary and interstellar dust observed by the Wind/WAVES electric field instrument, Geophys. Res. Lett., 41, 266-272, doi:10.1002/2013GL058786.

Mann, I., et al. (2011), Dusty plasma effects in near Earth space and interplanetary medium, Space Sci. Rev., 161, 1-47.

Mann, I., N. Meyer-Vernet, and A. Czechowski (2014), Dust in the planetary system: Dust interactions in space plasmas of the solar system, Phys. Rep., 536, 1-39.

McBride, N., and J. A. M. McDonnell (1999), Meteoroid impacts on spacecraft: Sporadics, streams, and the 1999 Leonids, Planet. Space Sci., 47, 1005-1013.

Meuris, P., N. Meyer-Vernet, and J. F. Lemaire (1996), The detection of dust grains by a wire dipole antenna: The radio dust analyzer, J. Geophys. Res., 101, 24,471-24,477.

Meyer-Vernet, N. (1985), Comet Giacobini-Zinner diagnosis from radio measurements, Adv. Space Res., 5, 37-46.

Meyer-Vernet, N., M. G. Aubier, and B. M. Pedersen (1986a), Voyager 2 at Uranus: Grain impacts in the ring plane, Geophys. Res. Lett., 13, 617-620.

Meyer-Vernet, N., et al. (1986b), Plasma diagnosis from thermal noise and limits on dust flux or mass in Comet Giacobini-Zinner, Science, 232, 370-374.

Meyer-Vernet, N., and C. Perche (1989), Toolkit for antennae and thermal noise near the plasma frequency, J. Geophys. Res., 94, 2405-2415.

Meyer-Vernet, N., A. Lecacheux, and B. M. Pedersen (1996), Constraints on Saturn's E ring from the Voyager-1 radioastronomy instrument, Icarus, 123, 113-128.

Meyer-Vernet, N., A. Lecacheux, and B. M. Pedersen (1998), Constraints on Saturn's G ring from the Voyager-2 radioastronomy instrument, Icarus, 132, 311-320.

Meyer-Vernet, N., et al. (2009a), Voltage pulses on STEREO/WAVES: Nanoparticles picked-up by the solar wind? Sol. Phys., $256,463-474$.

Meyer-Vernet, N., A. Lecacheux, M. L. Kaiser, and D. A. Gurnett (2009b), Detecting nanoparticles at radio frequencies: Jovian dust stream impacts on Cassini/RPWS, Geophys. Res. Lett., 36, L03103, doi:10.1029/2008GL036752.

Moncuquet, M., and P. Schippers (2013), Eos Trans. AGU, XX, Fall Meet. Suppl., Abstract P32B-05.

Oberc, P. (1996), Electric antenna as a dust detector, Adv. Space Res., 17, (12)105-(12)110.

Pantellini, F., S. Belheouane, N. Meyer-Vernet, and A. Zaslavsky (2012), Nano dust impacts on spacecraft and boom antenna charging, Astrophys. Space Sci., 341, 309-314.

Pantellini, F., et al. (2013), On the detection of nano dust using spacecraft based boom antennas, in Solar Wind 13 AIP Conference Proceedings, vol. 1539, edited by G. P. Zank et al., pp. 414-417, American Institute of Physics, Melville, N. Y.

Pedersen, B. M., et al. (1991), Dust distribution around Neptune: Grain impacts near the ring plane measured by the Voyager radioastronomy experiment, J. Geophys. Res., 96, 19,187-19,196.

Schippers, P., M. Moncuquet, N. Meyer-Vernet, and A. Lecacheux (2013), Core electron temperature and density in the innermost Saturn's magnetosphere from HF power spectra analysis on Cassini, J. Geophys. Res. Space Physics, 118, 7170-7180, doi:10.1002/2013JA019199.

Sitruk, L., and R. Manning (1997), L'experience spatiale G.G.S./Wind/WAVES: Ondes radio lectriques et ondes de plasma, CDPP Technical Document.

Zaslavsky, A., et al. (2012), Interplanetary dust detection by radio antennas: Mass calibration and fluxes measured by STEREO/WAVES, J. Geophys. Res., 117, A05102, doi:10.1029/2011JA017480. 